\title{
Research on Internet Information Security in the Big Data Era
}

\author{
Yang Shen ${ }^{1}$ \\ ${ }^{1}$ Dalian Vocational \& Technical College, Dalian, Liaoning, China
}

\begin{abstract}
In the era of big data, due to the great influence of big data itself, Internet information security has also become the focus of attention. In order to avoid disturbing people's lives, this article summarizes the opportunities and challenges in the era of big data based on previous work experience. This article analyzes and studies five aspects including establishing complete laws and regulations, protecting personal information, applying big data technology to public security systems, doing a good job in data management and classification, and ensuring the security of data transmission. The author discusses specific measures for the maintenance of Internet information security in the era of big data from the above five aspects.
\end{abstract}

\section{Introduction}

In general, with the continuous development and advancement of big data, the world is developing rapidly in the direction of informatization and science. Under the influence of big data, people's lives have become more convenient, and social information spread faster and faster. It can also be seen from the development of various industries that enterprises have gradually realized in-depth data analysis and resource sharing through their own exploration and application of big data. This can make development work in related fields more convenient, clarify the development goals of each company, and keep the company in a state of rapid development.

\section{Overview of Internet Big Data}

\subsection{The Meaning of Big Data}

At the present stage, people often say that big data represents a collection of data. In daily work, people will use these data to provide guidance for follow-up work. Under normal circumstances, the management information system in the big data environment is mainly collected by management personnel through computer software, hardware and network. Subsequently, big data can be transmitted and processed to get the type of data people want. With the help of the above operations, the overall business efficiency can be substantially improved. In addition, with the continuous development of computer technology, people's demand for computer technology has become more and more obvious. Especially in the big data environment, many enterprise managers began to pay attention to the data collection of the management information system, and realized the integration of data through various means to show higher value.

\subsection{Characteristics of Big Data}

From the comparison of big data and traditional databases, it can be seen that the most obvious characteristics are concentrated in the following aspects. Firstly, the database storage capacity is relatively large. The storage capacity of a big data database is several times higher than that of a traditional database. Secondly, big data has diverse characteristics. Except to storing text information, big data can also store information such as videos and images. Thirdly, big data has obvious high-speed characteristics. The so-called high speed of big data mainly refers to the ability of high-speed operation to meet people's actual needs for data in a short time. Fourth, big data is volatile. Big data itself has a multi-layer structure, which also means that big data has many forms and types. Figure 1 shows the application characteristics of big data in online marketing. Compared with traditional business data, it can be seen that big data itself has obvious vague and irregular characteristics, and it is difficult for traditional application software to analyze it comprehensively. Therefore, the effect of traditional software is very limited. 


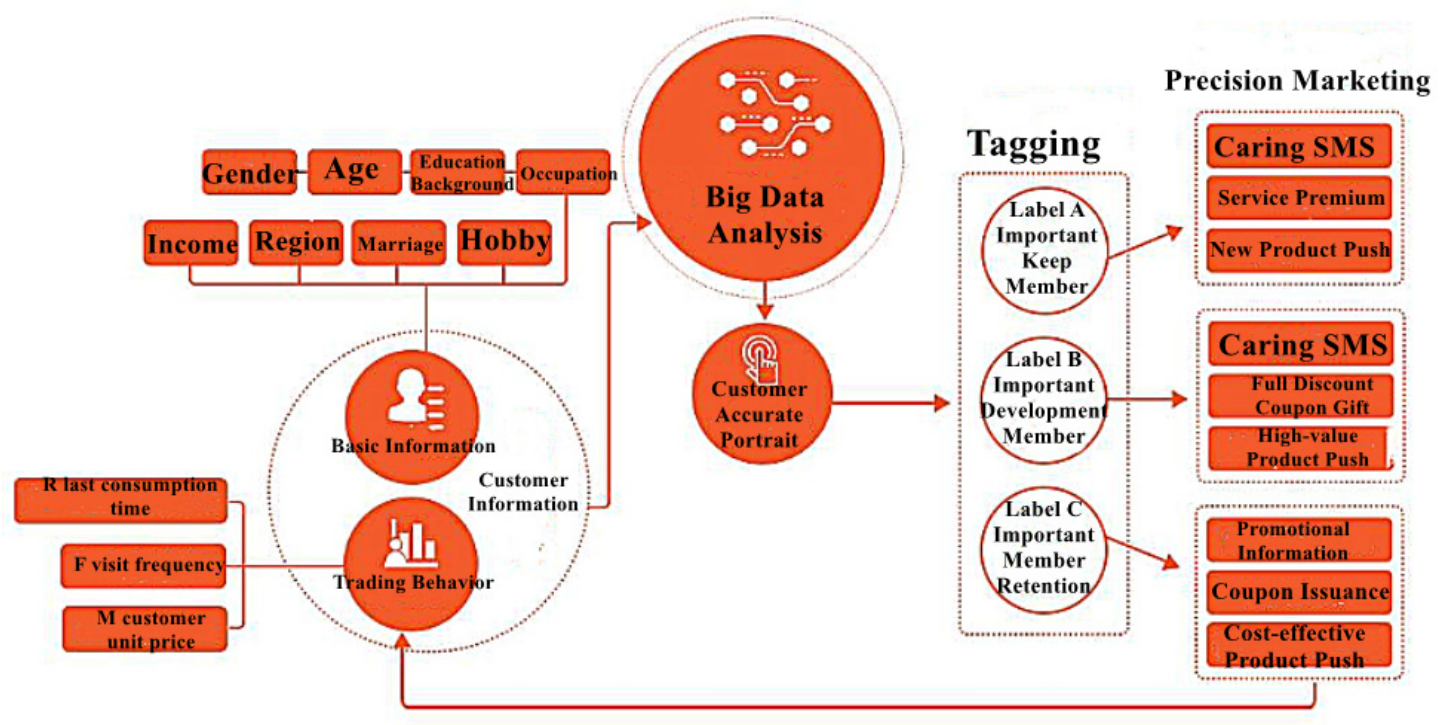

Figure 1 Application Characteristics of Big Data in Online Marketing

\section{Opportunities and Challenges in the Era of Big Data}

\subsection{Opportunities}

Under the influence of big data, the world has undergone earth-shaking changes, and the development speed of various fields has also increased. Hence, people need to face up to the opportunities presented by the era of big data. First, people can break through the shackles of traditional data and obtain more new resource data. The actual data acquisition methods will also become more diverse and convenient, and the data acquisition time will be greatly shortened. For example, many data sample processing procedures are involved in the process of visual analysis. The visual characteristics of the data can make the characteristics of the data sample more intuitive, which is conducive to users' in-depth understanding of the sample data and makes people more accepting of the data. What we need to pay attention to is that simple visual analysis cannot be effective in the face of massive data, which leads to a significant decline in user acceptance of samples. With the continuous development of science and technology, big data integrates traditional statistics and methods to make statistical work faster. For example, the application of technologies such as computers and learning algorithms has evolved many new technologies. Besides, with the continuous development of information technology, the threshold for big data applications has dropped. This has also increased the reliance on this technology in fields such as artificial intelligence and commerce, bringing more opportunities for the development of enterprises and society.

\subsection{Challenges}

Generally speaking, information and networks are always open and shared. In this way, many data and information resources are easily attacked, and corresponding Internet information security problems have evolved. Such problems are also one of the main sources of Internet hidden dangers. At present, people are very concerned about information security issues, but there is no effective measure to avoid them all. Except to enjoying the various conveniences brought by information technology, people will also expose their own information in the public eye. This situation is an unavoidable problem at this stage. Relevant statistics show that the current number of Internet users in China has exceeded 800 million. Among them, more than 50\% of WeChat users. People are obsessed with network information while forgetting the hidden dangers that Internet information security brings to people, and people's awareness of prevention is seriously insufficient.

\section{The Main Content of Internet Information Security Protection}

To truly achieve effective protection of Internet information security, people need to grasp the key points of information input and do a good job of protecting the data transmission entrance. Only in this way can we truly reduce the probability of information security problems. The most common information portals include websites, servers, etc. As a result, compared with traditional information security protection measures, new protection technologies must be more specific and mature, and the scope of their involvement must be broader. Moreover, it can present website and server protection functions. It should be noted that many current information protection methods cannot be cut off from traditional information security technologies, and firewall technology is still needed to make the protection work more complete. In addition, people will also introduce anti-virus software and hacker intrusion detection devices to prevent the integrity of the data path from being affected. It can also be seen from this that traditional information security protection technology and related measures play a very 
important role in the current protection work, and we need to achieve layered detection and prevention. Only in this way can the probability of information leakage problems be reduced. It can also be seen from this that China is unable to conduct in-depth research on information security technology at this stage, and the efficiency of problem solving is also very limited. In consequence, in the context of the big data era, the technical issues of information network security cannot be ignored.

\section{Specific Measures for Internet Information Security Maintenance in the Era of Big Data}

The improvement of big data technology can not only contribute to social development, but also bring some new problems. With the increase in the amount of global information, countries need to improve the completeness of information processing technology as soon as possible. Only in this way can we keep pace with the requirements of the big data era and make the world's science and technology advance steadily.

\subsection{Establish Sound Laws and Regulations}

In recent years, there have been more and more personal information leakage incidents on the Internet. In order to restrain this phenomenon, China promulgated the latest Cybersecurity Law of the People's Republic of China in 2016, which clearly stated that information resources are of great significance in China's overall economic development and construction. Only by grasping the abundant data and information can the country's competitiveness be kept at a higher level. Although China has made great efforts in the development and management of network information technology, there are no corresponding laws and regulations at this stage. As a consequence, relevant departments should start from many aspects such as big data technology management and security, and formulate more complete laws and regulations. This is also the foundation of maintaining network information security.

\subsection{Protect Personal Information}

China formulated and officially implemented the "Guidelines for the Protection of Personal Information in Information Security Technology Public and Commercial Service Information Systems" on February 1, 2013. However, there are still many problems in the actual application of this guide, and we cannot keep personal information in a good state. More importantly, some concepts and subjects have never been fully perfected and clarified, and the penalties for violations are seriously insufficient. Most consumers cannot use this system to protect their legitimate rights and interests. As of June 2020, China's online education users have reached 381 million. Chinese netizens accounted for $40.5 \%$ of the total netizens, the number of online medical users was 276 million, accounting for $29.4 \%$ of the total netizens, and the number of remote office users was 199 million, accounting for $21.2 \%$ of the total netizens. This is also the main process of the power of the network. one. To this end, China can learn from the development experience of developed countries, and should impose severe penalties and substantially increase the amount of fines in the event of violations of citizens' personal information, such as leaking or reselling personal information. Meanwhile, we should also include this behavior in the personal integrity file to restrict many subsequent economic activities and resolutely punish illegal behaviors.

\subsection{Apply Big Data Technology to Public Security System}

Public security organs should change their previous work concepts in the course of their work, and appropriately innovate their thinking in handling cases. First of all, public security personnel must be aware of the importance of big data technology and integrate this technology with the case-handling process. The implementation of in-depth decision-making and research operations can improve the technical work standards of public security officers. Secondly, people must also establish complete data information standards in the public security system to clarify the content of the data system. Only in this way can it be ensured that the public security organs have more resources, show a greater role, ensure information sharing among different departments, and save case-handling resources. Thirdly, the public security organs must also be aware of the importance of information collection. Only by ensuring the abundance of information can the effectiveness of case handling be enhanced. Otherwise the staff should focus on the maintenance of the quality of information collection and formulate higher data collection standards. In the meantime, staff also need to establish cooperative relations with other departments to truly share resources. Fourth, public security personnel should strengthen their own learning capabilities and learn more about big data technology. In order to ensure the security of data in the application of big data, the communication between nodes and between the client and the server requires appropriate encryption measures. Due to the different communication methods, the encryption forms involved are also quite different. People can also isolate the direct access of the client and the big data platform through the gateway server, so that the system is always in a safe state. Relevant departments should also establish gateway servers to ensure data security through various measures such as identity authentication and access control.

\subsection{Do A Good Job in Data Management and Classification}

From the actual development and research of big data, it can be seen that there are many types and huge amounts of information in the current database, which further 
reduces the density of data value. The staff want to select valuable information from the massive data, in addition to ensuring the security and practicality of data applications, but also to continue the data convenience and time-saving characteristics. Therefore, effective data management should be implemented in the extraction of large amounts of information and data, and the data should be fully classified to create more favorable conditions for later use. For the formulation of the management system and structure for a large number of households, the staff should set different access rights for different users to ensure more detailed division of rights. Furthermore, staff can target two types of objects, user groups and individual users, to make the authority design more detailed. Finally, the staff should pay attention to strengthening the system security operation in addition to the authority design, and set up security mechanisms such as firewalls.

\subsection{Ensure the Security of Data Transmission}

In the application of data information, a series of problems such as data transmission and input will occur. In order to reduce the occurrence of the above problems, the implementation of encryption and protection is very important. For a series of encryption programs and software in the equipment, we should formulate reasonable protection measures to reduce the probability and risk of data leakage. In the later stage, if people want to obtain data information, they should first decrypt the data before they can continue to use the data. Whether it is data management, data classification, user authority design and system security, protection and encryption should be done during the entire data transmission process. The actual data transmission port design is mainly based on encryption and decryption procedures. Increase the connection with the system account, setting the key can make the entire data transmission more secure.

\section{Transformation of Information Security Management}

Big data itself has obvious interactivity and sharing characteristics, which puts forward a major test for the setting of Internet information security management mode. Under the traditional information security technology management model, generally only a single individual and a single organization are involved, or the two are combined to monitor and present related security risks. However, due to the influence of big data, many data types such as multi-dimensional and multi-form data can be integrated in a short time. Coupled with the influence of information technology such as the Internet, data can be mined from different angles. At this time, the intrinsic value of the data can also be presented. Simultaneously, because the data itself has the characteristics of openness and transparency, the problems presented are more obvious, and the source of its exposure will become more accurate and specific. For how to change traditional security technologies, people need to be aware of the driving and supporting role of big data core technologies, break through the limitations of traditional technologies in the past, and demonstrate higher value.

\section{Conclusion}

In summary, as the application of big data technology becomes more and more widespread, the Internet has appeared in people's lives. While the Internet has brought many benefits to people, it has also created some new problems. In this process, the security issue is particularly prominent. As a result, people should realize that big data is a double-edged sword. We need to seriously think about the various problems that exist, and properly solve them to play a greater role.

\section{Acknowledgments}

Liaoning Province scientific research funding projects in 2019:Research on the Application of Big Data Technology in E-commerce Platform (JYT201904)

\section{References:}

1. Fan Xing. Research on Internet Information Security in the Big Data Era[J]. Network Security Technology and Application, 2020(09): 55-57.

2. Liu Xiaohua. Research on Internet Finance Innovation in the Era of Big Data[J]. Financial Technology Era, 2020(09): 94-96.

3. Zhang Chaohui. Opportunities and challenges facing accounting theory in the era of "Internet + " big data[J]. Accounting Study, 2020(18): 114-115.

4. Liu Shiyun, Song Haibo. Research on Computer Network Security Technology in the Data Age[J]. Wireless Internet Technology, 2019, 16(21): 126-127.

5. Zhang Tingyu. The influence of Internet big data on accounting work[J]. Modern Economic Information, 2019(18): $156+158$. 